\title{
Stabilizing Transmission Intervals for Networked Control Systems with Nonlinear Delay Dynamics
}

\author{
Domagoj Tolić and Sandra Hirche
}

\begin{abstract}
In this paper, we consider a nonlinear delay process to be controlled over a communication network in the presence of disturbances and study robustness of the resulting closed-loop system with respect to network-induced phenomena such as sampled, distorted and delayed data as well as scheduling protocols. Maximally Allowable Transfer Interval (MATI) labels the greatest transmission interval for which a prescribed $\mathcal{L}_{p}$-gain, as a measure of control performance, is attained. The proposed methodology combines impulsive delay system modeling with Lyapunov-Razumikhin techniques to allow for communication delays greater than MATIs. Other salient features of our methodology are the consideration of nonuniform variable delays and employment of model-based estimators to prolong MATIs. The present stability results are provided for the class of Uniformly Globally Exponentially Stable (UGES) scheduling protocols such as Round Robin (RR) and Try-Once-Discard (TOD). Finally, a nonlinear example is provided to demonstrate the benefits of our methodology.
\end{abstract}

\section{INTRODUCTION}

Networked Control Systems (NCSs) are spatially distributed systems for which the communication between sensors, actuators and controllers is realized by a shared (wired or wireless) communication network [1]. In comparison with conventional control systems in which parts of control loops exchange information via dedicated point-topoint connections, NCSs offer several advantages such as reduced installation and maintenance costs as well as greater flexibility. However, NCSs also generate imperfections (e.g., sampled, corrupted and delayed data) that impair the control system performance and can even lead to instability. In addition, scheduling protocols might govern the communication medium access due to channel capacity constraints or to reduce packet collisions among uncoordinated NCS links. Since the aforementioned network-induced phenomena occur simultaneously, the investigation of their cumulative adverse effects on the NCS performance is of particular interest.

In this paper, we consider a nonlinear delay system to be controlled by a nonlinear delay dynamic controller

D. Tolić is with Faculty of Electrical Engineering and Computing, University of Zagreb, Unska 3, 10000 Zagreb, Croatia. S. Hirche is with the Chair of Information-oriented Control, Technische Universität München, Arcisstraße 21, D-80290 München, Germany. domagoj.tolic@fer.hr, hirche@tum.de

This work has been supported by the European Community Seventh Framework Programme under grant No. 285939 (ACROSS). D. Tolić is partially supported by the Air Force Research Laboratory under agreement number FA8655-13-1-3055.

The U.S. Government is authorized to reproduce and distribute reprints for Governmental purposes notwithstanding any copyright notation thereon. The views and conclusions contained herein are those of the authors and should not be interpreted as necessarily representing the official policies or endorsements, either expressed or implied, of the Air Force Research Laboratory or the U.S. Government. over a communication network in the presence of exogenous/modeling disturbances, scheduling protocols among NCS links, time-varying signal delays, time-varying transmission intervals and distorted data. Besides networkinduced delays, notice that delays might be present in the plant and controller dynamics as well. Hence the term delay NCSs. The present paper extends the emulation-based approach from [2] towards plants and controllers with delay dynamics as well as towards nonuniform time-varying NCS link delays. In other words, different NCS links induce different nonconstant delays. To the best of our knowledge, the work presented herein is the most comprehensive study of the aforementioned cumulative effects as far as the actual plant-controller dynamics (i.e., time-varying, nonlinear, delayed and with disturbances) and interconnection (i.e., output feedback) as well as the variety of scheduling protocols (i.e., UGES protocols) and other network-induced phenomena are concerned (i.e., variable delays, communication channels with distortions). For instance, [3] focuses on time-varying control affine plants (i.e., no delay dynamics in the plant nor controller) and state feedback with a constant delay whilst neither exogenous/modeling disturbances, distorted data nor scheduling protocols are taken into account. Similar comparisons can be drawn with respect to other related works (see [1]-[6] and the references therein).

Our methodology employs impulsive delay system modeling and Lyapunov-Razumikhin techniques when computing Maximally Allowable Transmission Intervals (MATIs) that provably stabilize NCSs for the class of Uniformly Globally Exponentially Stable (UGES) scheduling protocols. Besides merely stabilizing MATIs, our methodology also designs MATIs that yield a prespecified level of control system performance in terms of $\mathcal{L}_{p}$-gains. Delay system modeling (rather than ODE modeling as in [4]) allows us to employ model-based estimators in order to increase MATIs [7]. In addition, real-life applications are characterized by corrupted data due to, among others, measurement noise and communication channel distortions. In order to include distorted information (in addition to exogenous/modeling disturbances) into the stability analyses, we use the notion of $\mathcal{L}_{p}$-stability with bias.

The main contributions of this paper are fourfold: a) the design of MATIs in nonlinear delay NCSs with UGES protocols even for the so-called large delays; b) the LyapunovRazumikhin-based procedure for rendering $\mathcal{L}_{p}$-stability of nonlinear impulsive delay systems and computing the associated $\mathcal{L}_{p}$-gains; c) the consideration of NCS links with nonidentical time-dependent delays; and d) the inclusion of 
model-based estimators. In contrast to [2], this paper incorporates nonuniform variable delays, plants/controllers with delay dynamics, provides a nonlinear numerical example and designs model-based estimation that prolongs MATIs.

The remainder of this paper is organized as follows. Section II presents the utilized notation and stability notions regarding impulsive delay systems. Section III states the problem of finding MATIs for nonlinear delay NCSs with UGES protocols in the presence of nonuniform communication delays and exogenous/modeling disturbances. A methodology to solve the problem is presented in Section IV. A numerical example is provided in Section V. Conclusions and future challenges are in Section VI.

\section{PRELIMinaries}

\section{A. Notation}

To simplify notation, we use $(x, y):=\left[\begin{array}{ll}x^{\top} & y^{\top}\end{array}\right]^{\top}$. The dimension of a vector $x$ is denoted $n_{x}$. Next, let $f: \mathbb{R} \rightarrow \mathbb{R}^{n}$ be a Lebesgue measurable function on $[a, b] \subset \mathbb{R}$. We use

$$
\|f[a, b]\|_{p}:=\left(\int_{[a, b]}\|f(s)\|^{p} \mathrm{~d} s\right)^{\frac{1}{p}}
$$

to denote the $\mathcal{L}_{p}$-norm of $f$ when restricted to the interval $[a, b]$. If the corresponding norm is finite, we write $f \in$ $\mathcal{L}_{p}[a, b]$. In the above expression, $\|\cdot\|$ refers to the Euclidean norm of a vector. If the argument of $\|\cdot\|$ is a matrix $A$, then it denotes the induced 2-norm of $A$. Furthermore, $|\cdot|$ denotes the (scalar) absolute value function. The $n$ dimensional vector with all zero entries is denoted $\mathbf{0}_{n}$. In addition, $\mathbb{R}_{+}^{n}$ denotes the nonnegative orthant. The natural numbers are denoted $\mathbb{N}$ or $\mathbb{N}_{0}$ when zero is included.

Left-hand and right-hand limits are denoted $x\left(t^{-}\right)=$ $\lim _{t^{\prime} \nearrow_{t}} x\left(t^{\prime}\right)$ and $x\left(t^{+}\right)=\lim _{t^{\prime} \searrow t} x\left(t^{\prime}\right)$, respectively. Next, for a set $\mathcal{S} \subseteq \mathbb{R}^{n}$, let $\operatorname{PC}([a, b], \mathcal{S})=\{\phi:[a, b] \rightarrow$ $\mathcal{S} \mid \phi(t)=\phi\left(t^{+}\right)$for every $t \in[a, b), \phi\left(t^{-}\right)$exists in $\mathcal{S}$ for all $t \in(a, b]$ and $\phi\left(t^{-}\right)=\phi(t)$ for all but at most a finite number of points $t \in(a, b]\}$. Observe that $\operatorname{PC}([a, b], \mathcal{S})$ denotes the family of right-continuous functions on $[a, b)$ with finite left-hand limits on $(a, b]$ contained in $S$ and whose discontinuities do not accumulate in finite time.

\section{B. Impulsive Delay Systems}

In this paper, we consider nonlinear impulsive delay systems

$$
\Sigma\left\{\begin{aligned}
\chi\left(t^{+}\right) & =h_{\chi}\left(t, \chi_{t}\right) \\
\dot{\chi}(t) & =f_{\chi}\left(t, \chi_{t}, \omega\right) \\
y & =\ell_{\chi}\left(t, \chi_{t}, \omega\right)
\end{aligned}\right\} \quad t \in \mathcal{T}
$$

where $\chi \in \mathbb{R}^{n_{\chi}}$ is the state, $\omega \in \mathbb{R}^{n_{\omega}}$ is the input and $y \in \mathbb{R}^{n_{y}}$ is the output. The functions $f_{\chi}$ and $h_{\chi}$ are regular enough to guarantee forward completeness of solutions which, given initial time $t_{0}$ and initial condition $\chi_{t_{0}} \in$ $P C\left([-d, 0], \mathbb{R}^{n_{\chi}}\right)$, where $d \geq 0$ is the maximum value of all time-varying delay phenomena, are given by right-continuous functions $t \mapsto \chi(t) \in P C\left(\left[t_{0}-d, \infty\right], \mathbb{R}^{n_{\chi}}\right)$. Furthermore, $\chi_{t}$ denotes the translation operator acting on the trajectory $\chi(\cdot)$ defined by $\chi_{t}(\theta):=\chi(t+\theta)$ for $-d \leq \theta \leq 0$. In other words, $\chi_{t}$ is the restriction of trajectory $\chi(\cdot)$ to the interval $[t-d, t]$ and translated to $[-d, 0]$. For $\chi_{t} \in P C\left([-d, 0], \mathbb{R}^{n_{\chi}}\right)$, the norm of $\chi_{t}$ is defined by $\left\|\chi_{t}\right\|=\sup _{-d \leq \theta \leq 0}\left\|\chi_{t}(\theta)\right\|$. Jumps of the state are denoted $\chi\left(t^{+}\right)$and occur at time instants $t \in \mathcal{T}:=\left\{t_{1}, t_{2}, \ldots\right\}$, where $t_{i}<t_{i+1}, i \in \mathbb{N}_{0}$. The value of the state after a jump is given by $\chi\left(t^{+}\right)$for each $t \in \mathcal{T}$. For a comprehensive discussion regarding the solutions to (1) considered herein, refer to [8, Chapter $2 \& 3$ ].

Definition $1\left(\mathcal{L}_{p}\right.$-Stability with Bias $\left.b\right)$ : Let $p \in[1, \infty]$. The system $\Sigma$ is $\mathcal{L}_{p}$-stable with bias $b(t) \equiv b \geq 0$ from $\omega$ to $y$ with (linear) gain $\gamma \geq 0$ if there exists $K \geq 0$ such that, for each $t_{0} \in \mathbb{R}$ and each $\chi_{t_{0}} \in P C\left([-d, 0], \mathbb{R}^{n_{\chi}}\right)$, each solution to $\Sigma$ from $\chi_{t_{0}}$ satisfies $\left\|y\left[t_{0}, t\right]\right\|_{p} \leq K\left\|\chi_{t_{0}}\right\|+$ $\gamma\left\|\omega\left[t_{0}, t\right]\right\|_{p}+\left\|b\left[t_{0}, t\right]\right\|_{p}$ for each $t \geq t_{0}$.

Definition 2 ( $\mathcal{L}_{p}$-Detectability): Let $p \in[1, \infty]$. The state $\chi$ of $\Sigma$ is $\mathcal{L}_{p}$-detectable from $(y, \omega)$ with (linear) gain $\gamma \geq$ 0 if there exists $K \geq 0$ such that, for each $t_{0} \in \mathbb{R}$ and each $\chi_{t_{0}} \in P C\left([-d, 0], \mathbb{R}^{n_{\chi}}\right)$, each solution to $\Sigma$ from $\chi_{t_{0}}$ satisfies $\left\|\chi\left[t_{0}, t\right]\right\|_{p} \leq K\left\|\chi_{t_{0}}\right\|+\gamma\left\|y\left[t_{0}, t\right]\right\|_{p}+\gamma\left\|\omega\left[t_{0}, t\right]\right\|_{p}$ for each $t \geq t_{0}$.

When $b=0$, we say " $\mathcal{L}_{p}$-stability" instead of " $\mathcal{L}_{p}$-stability with bias 0 ".

\section{Problem Statement}

Consider a nonlinear plant with delay dynamics

$$
\begin{aligned}
\dot{x}_{p} & =f_{p}\left(t, x_{p_{t}}, u, \omega_{p}\right), \\
y & =g_{p}\left(t, x_{p_{t}}\right),
\end{aligned}
$$

and a controller with delay dynamics

$$
\begin{aligned}
\dot{x}_{c} & =f_{c}\left(t, x_{c_{t}}, u_{c}, \omega_{c}\right), \\
y_{c} & =g_{c}\left(t, x_{c_{t}}\right),
\end{aligned}
$$

interconnected via the assignment

$$
y_{c}=u, \quad y=u_{c}
$$

where $x_{p} \in \mathbb{R}^{n_{p}}$ and $x_{c} \in \mathbb{R}^{n_{c}}$ are the states, $y \in \mathbb{R}^{n_{y}}$ and $u \in \mathbb{R}^{n_{u}}$ are the outputs, and $\left(u, \omega_{p}\right) \in \mathbb{R}^{n_{u}} \times \mathbb{R}^{n_{\omega_{p}}}$ and $\left(y, \omega_{c}\right) \in \mathbb{R}^{n_{y}} \times \mathbb{R}^{n_{\omega_{c}}}$ are the inputs of the plant and controller, respectively, where $\omega_{p}$ and $\omega_{c}$ are external disturbances to (and/or modeling uncertainties of) the plant and controller, respectively. The translation operators $x_{p_{t}}$ and $x_{c_{t}}$ are defined in Section II-B while the corresponding plant and controller delays are $d_{p} \geq 0$ and $d_{c} \geq 0$, respectively. For notational convenience, constant plant and controller delays are considered.

Let us now model the communication network between the plant and controller over which intermittent exchange of information takes place (see Figure 1). The value of $u$ computed by the controller that arrives at the plant is denoted $\hat{u}$. Similarly, the values of $y$ that the controller actually receives are denoted $\hat{y}$. We proceed further by defining the error vector

$$
e=\left[\begin{array}{l}
e_{y}(t) \\
e_{u}(t)
\end{array}\right]:=\left[\begin{array}{c}
\hat{y}(t)-y_{t} \\
\hat{u}(t)-u_{t}
\end{array}\right]
$$




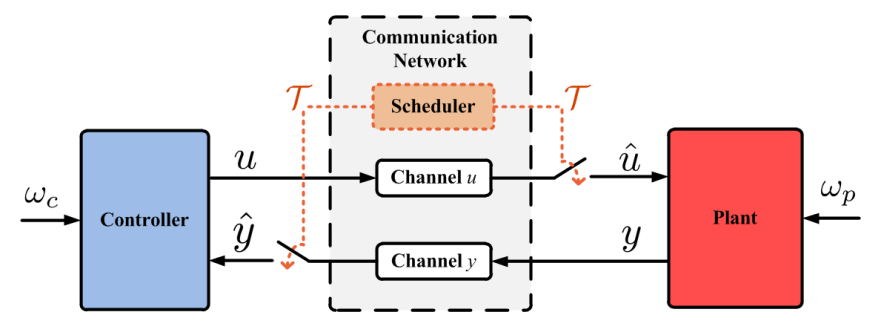

Fig. 1. A plant and controller interacting over a communication network. The switches indicate that the information between the plant and controller are exchanged (complying with some scheduling protocol among the NCS links) at discrete time instants belonging to a set $\mathcal{T}$. The communication delays in each NCS link are time varying and different.

where $y_{t}$ and $u_{t}$ are translation operators and the maximal network-induced delay $d \geq 0$ (e.g., propagation delays and/or delays arising from protocol arbitration). The operator $\left(y_{t}, u_{t}\right)$ in (5) delays each component of $(y, u)$ for the respective delay. Essentially, if the $i^{\text {th }}$ component of $(y(t), u(t))$, that is $(y(t), u(t))_{i}$, is transmitted with delay $d_{i}: \mathbb{R} \rightarrow \mathbb{R}_{+}$, then the $i^{\text {th }}$ components of $\left(y_{t}, u_{t}\right)$, that is $\left(y_{t}, u_{t}\right)_{i}$, is in fact $\left(y\left(t-d_{i}(t)\right), u\left(t-d_{i}(t)\right)\right)_{i}$. Accordingly, $d:=\max \left\{\sup _{t \in \mathbb{R}} d_{1}(t), \ldots, \sup _{t \in \mathbb{R}} d_{n_{y}+n_{u}}(t)\right\}$.

Due to intermittent transmissions of the components of $y$ and $u$, the respective components of $\hat{y}$ and $\hat{u}$ are updated at time instants $t_{1}, t_{2}, \ldots, t_{i}, \ldots \in \mathcal{T}$, i.e.,

$$
\left.\begin{array}{l}
\hat{y}\left(t_{i}^{+}\right)=y_{t}+h_{y}\left(t_{i}, e\left(t_{i}\right)\right) \\
\hat{u}\left(t_{i}^{+}\right)=u_{t}+h_{u}\left(t_{i}, e\left(t_{i}\right)\right)
\end{array}\right\} \quad t_{i} \in \mathcal{T},
$$

where $h_{y}: \mathbb{R} \times \mathbb{R}^{n_{e}} \rightarrow \mathbb{R}^{n_{y}}$ and $h_{u}: \mathbb{R} \times \mathbb{R}^{n_{e}} \rightarrow \mathbb{R}^{n_{u}}$ model measurement noise, channel distortion and the underlying scheduling protocol. The role of $h_{y}$ and $h_{u}$ is as follows. Suppose that the NCS has $l$ links. Accordingly, the error vector $e$ can be partitioned $e:=\left(e_{1}, \ldots, e_{l}\right)$. In order to avoid cumbersome indices, let us assume that each NCS link is characterized by its own delay. Hence, there are merely $l$ (rather than $n_{y}+n_{u}$ ) different delays $d_{i}: \mathbb{R} \rightarrow \mathbb{R}_{+}$in (5). Besides the already introduced upper bound $d$ on $d_{i}(t)$ 's, we assume that $d_{i}(t)$ 's are differentiable with bounded $\left|\dot{d}_{i}(t)\right|$. As orchestrated by (6), if the $j^{\text {th }}$ NCS link is granted access to the communication medium at some $t_{i} \in \mathcal{T}$, the corresponding components of $\left(\hat{y}\left(t_{i}\right), \hat{u}\left(t_{i}\right)\right)$ jump to the received values. It is to be noted that all other components of $\left(\hat{y}\left(t_{i}\right), \hat{u}\left(t_{i}\right)\right)$ remain unaltered. Consequently, the related components of $e\left(t_{i}\right)$ reset to the noise $\nu_{j}\left(t_{i}\right)$ present in the received data, i.e.,

$$
e_{j}\left(t_{i}^{+}\right)=\nu_{j}\left(t_{i}\right)
$$

and we assume that

$$
\sup _{t \in \mathbb{R}, j \in\{1, \ldots, l\}}\left\|\nu_{j}(t)\right\|=K_{\nu} .
$$

Noise $\nu_{j}\left(t_{i}\right)$, which is embedded in $h_{y}$ and $h_{u}$, models any discrepancy between the received values and their actual values at time $t_{i}-d_{j}(t)$ (when the $j^{\text {th }}$ NCS link of $(y(t), u(t)$ ) was sampled). We point out that $\nu_{j}$ has nothing to do with $\omega_{p}$ nor $\omega_{c}$.
In between transmissions, the values of $\hat{y}$ and $\hat{u}$ need not to be constant as in [4], but can be estimated in order to extend transmission intervals (consult [7] for more). In other words, for each $t \in\left[t_{0}, \infty\right) \backslash \mathcal{T}$ we have

$$
\begin{aligned}
& \dot{\hat{y}}=\hat{f}_{p}\left(t, x_{p_{t}}, x_{c_{t}}, \hat{y}_{t}, \hat{u}_{t}, \omega_{p}, \omega_{c}\right), \\
& \dot{\hat{u}}=\hat{f}_{c}\left(t, x_{p_{t}}, x_{c_{t}}, \hat{y}_{t}, \hat{u}_{t}, \omega_{p}, \omega_{c}\right) .
\end{aligned}
$$

The commonly used Zero-Order-Hold (ZOH) strategy is characterized by $\dot{\hat{y}} \equiv \mathbf{0}_{n_{y}}$ and $\dot{\hat{u}} \equiv \mathbf{0}_{n_{u}}$.

The following definition of UGES scheduling protocols is extracted from [9] and [4].

Definition 3: Consider the noise-free setting, i.e., $K_{\nu}=0$. The protocol given by $h:=\left(h_{y}, h_{u}\right)$ is UGES if there exists a function $W: \mathbb{N}_{0} \times \mathbb{R}^{n_{e}} \rightarrow \mathbb{R}_{+}$such that $W(i, \cdot): \mathbb{R}^{n_{e}} \rightarrow \mathbb{R}_{+}$ is locally Lipschitz (and hence almost everywhere differentiable) for every $i \in \mathbb{N}_{0}$, and if there exist positive constants $\underline{a}, \bar{a}$ and $0 \leq \rho<1$ such that

(i) $\underline{a}\|e\| \leq W(i, e) \leq \bar{a}\|e\|$, and

(ii) $W\left(i+1, h\left(t_{i}, e\right)\right) \leq \rho W(i, e)$,

for all $(i, e) \in \mathbb{N}_{0} \times \mathbb{R}^{n_{e}}$.

Commonly used UGES protocols are the Round Robin (RR) and Try-Once-Discard protocol (TOD) (consult [4], [9], [10]). The corresponding constants are $\underline{a}_{R R}=1, \bar{a}_{R R}=\sqrt{l}$, $\rho_{R R}=\sqrt{(l-1) / l}$ for $\mathrm{RR}$ and $\underline{a}_{T O D}=\bar{a}_{T O D}=1$, $\rho_{\text {TOD }}=\sqrt{(l-1) / l}$ for TOD.

The properties imposed on the NCS in Figure 1 are summarized in the following standing assumption.

Assumption 1: The jump times of the NCS links at the controller and plant end obey the underlying UGES scheduling protocol and occur at transmission instants belonging to $\mathcal{T}:=\left\{t_{1}, t_{2}, \ldots, t_{i}, \ldots\right\}$, where $\epsilon \leq t_{i+1}-t_{i} \leq \tau$ for each $i \in \mathbb{N}_{0}$ with $\epsilon>0$ arbitrarily small. The received data is corrupted by measurement noise and/or channel distortion. In addition, each NCS link is characterized by the networkinduced delay $d_{i}(t), i \in\{1, \ldots, l\}$.

The existence of a strictly positive $\tau$, and therefore the existence of $\varepsilon>0$, is demonstrated in Remark 2 .

A typical closed-loop system (2)-(3) might be robust (in the $\mathcal{L}_{p}$ sense according to (15)) only for some sets of $d_{i}(t)$, $i \in\{1, \ldots, l\}$. We refer to the family of such delay sets as the family of admissible delays and denote it $\mathcal{D}$. Next, given some admissible delays $d_{i}(t), i \in\{1, \ldots, l\}$, the maximal $\tau$ which renders $\mathcal{L}_{p}$-stability (with a desired gain) of the closed-loop system (2)-(3) is called MATI and is denoted $\bar{\tau}$.

Problem 1: Given admissible delays $d_{i}(t), i \in\{1, \ldots, l\}$, estimator (8) and the UGES protocol of interest, determine the MATI $\bar{\tau}$ to update components of $(\hat{y}, \hat{u})$ such that the NCS (2)-(3) is $\mathcal{L}_{p}$-stable with bias and a prespecified $\mathcal{L}_{p}$-gain for some $p \in[1, \infty]$.

\section{Methodology}

Along the lines of [9], we rewrite the closed-loop system (2)-(3) in the following form amenable for small-gain 
theorem (see [11, Chapter 5]) analyses:

$$
\left.\begin{array}{l}
\left.\begin{array}{l}
x\left(t^{+}\right)=x(t) \\
e\left(t^{+}\right)=h(t, e(t))
\end{array}\right\} \quad t \in \mathcal{T} \\
\dot{x}=f\left(t, x_{t}, e, \omega\right) \\
\dot{e}=g\left(t, x_{t}, e_{t}, \omega_{t}\right)
\end{array}\right\} \quad \text { otherwise, }
$$

where $x:=\left(x_{p}, x_{c}\right), \omega:=\left(\omega_{p}, \omega_{c}\right)$, and functions $f, g$ and $h$ are given by (10) and (11). We assume enough regularity on $f$ and $g$ to guarantee existence of the solutions on the interval of interest [8, Chapter 3]. Observe that differentiability of $d_{i}(t)$ 's and boundedness of $\left|\dot{d}_{i}(t)\right|$ play an important role in attaining regularity of $g$. For the sake of simplicity, our notation does not explicitly distinguish between translation operators with delays $d_{p}, d_{c}, d$ or $2 d$ in (10), (11) and in what follows. In this regard, we point out that the operators $x_{p_{t}}$ and $x_{c_{t}}$ are with delays $d_{p}$ and $d_{c}$, respectively, the operators $g_{p_{t}}$ and $g_{c_{t}}$ within $\hat{f}_{p}$ and $\hat{f}_{c}$ are with delay $2 d$ while all other operators are with delay $d$. In what follows we also use $\bar{d}:=2 d+\max \left\{d_{p}, d_{c}\right\}$, which is the maximum value of all delay phenomena in (11).

For future reference, the delay dynamics

$$
\begin{aligned}
& \left.x\left(t^{+}\right)=x(t) \quad\right\} \quad t \in \mathcal{T} \\
& \left.\dot{x}=f\left(t, x_{t}, e, \omega\right) \quad\right\} \text { otherwise, }
\end{aligned}
$$

are termed the nominal system $\Sigma_{n}$, and the impulsive delay dynamics

$$
\begin{array}{lll}
\left.e\left(t^{+}\right)=h(t, e(t)) \quad\right\} & t \in \mathcal{T} \\
\left.\dot{e}=g\left(t, x_{t}, e_{t}, \omega_{t}\right) \quad\right\} \quad \text { otherwise, }
\end{array}
$$

are termed the error system $\Sigma_{e}$. The remainder of our methodology interconnects $\Sigma_{n}$ and $\Sigma_{e}$ using appropriate outputs. Subsequently, the small-gain theorem is employed to infer $\mathcal{L}_{p}$-stability with bias.

\section{A. $\mathcal{L}_{p}$-Stability with Bias of Impulsive Delay LTI Systems}

Let us establish conditions on the transmission interval $\tau$ and delay $d(t)$ that yield $\mathcal{L}_{p}$-stability with bias for a class of impulsive delay LTI systems. Clearly, the results of this subsection are later on applied towards achieving $\mathcal{L}_{p}$-stability with bias and an appropriate $\mathcal{L}_{p}$-gain of $\Sigma_{e}$.

Consider the following class of impulsive delay LTI system

$$
\begin{aligned}
\dot{\xi}(t) & =a \xi(t-d(t))+\tilde{u}(t), & & t \notin \mathcal{T} \\
\xi\left(t^{+}\right) & =c \xi(t)+\tilde{\nu}(t), & & t \in \mathcal{T},
\end{aligned}
$$

where $a \in \mathbb{R}$ and $c \in(-1,1)$, initialized with some $\xi_{t_{0}} \in$ $P C([-\breve{d}, 0], \mathbb{R})$. In addition, $d(t)$ is a continuous function upper bounded by $\breve{d}$ while $\tilde{u}, \tilde{\nu}: \mathbb{R} \rightarrow \mathbb{R}$ denote external inputs and $\tilde{\nu} \in \mathcal{L}_{\infty}$.

Lemma 1: Assume $\tilde{u} \equiv 0, \tilde{\nu} \equiv 0$ and consider a positive constant $r$. In addition, let $\lambda_{1}:=\frac{a^{2}}{r}$, and $\lambda_{2}:=c^{2}$ for $c \neq 0$ or merely $\lambda_{2} \in(0,1)$ for $c=0$. If there exist constants $\lambda>0, M>1$ such that the conditions

(I) $\tau\left(\lambda+r+\lambda_{1} M e^{-\lambda \tau}\right)<\ln M$, and
(II) $\tau\left(\lambda+r+\frac{\lambda_{1}}{\lambda_{2}} e^{\lambda \breve{d}}\right)<-\ln \lambda_{2}$

hold, then the system (14) is UGES and $\|\xi(t)\| \leq$ $\sqrt{M}\left\|\xi_{t_{0}}\right\| e^{-\frac{\lambda}{2}\left(t-t_{0}\right)}$ for all $t \geq t_{0}$.

The previous lemma, combined with the work presented in [12], results in the following theorem.

Theorem 1: Suppose that the system given by (14) is UGES with constants $\lambda>0$ and $M>1$ and that $\sup _{t \in \mathbb{R}}\|\tilde{\nu}(t)\| \leq \tilde{K}_{\nu}$. Then, the system (14) is $\mathcal{L}_{p}$-stable with bias $\frac{\tilde{K}_{\nu} \sqrt{M}}{e^{\frac{\lambda \epsilon}{2}}-1}$ from $\tilde{u}$ to $\xi$ and with gain $\frac{2}{\lambda} \sqrt{M}$ for each $p \in[1, \infty]$.

\section{B. Obtaining MATIs via the Small-Gain Theorem}

We now state the main result of this paper. Essentially, we interconnect $\Sigma_{n}$ and $\Sigma_{e}$ via suitable outputs (i.e., $H$ and $W$, respectively), impose the small-gain condition and invoke the small-gain theorem.

Theorem 2: Suppose the underlying UGES protocol, $d_{1}(t), \ldots, d_{l}(t)$ and $K_{\nu} \geq 0$ are given. In addition, assume that

(a) there exists a continuous function $H$ : $P C\left([-\bar{d}, 0], \mathbb{R}^{n_{x}}\right) \times P C\left([-d, 0], \mathbb{R}^{n_{\omega}}\right) \rightarrow \mathbb{R}^{m}$ such that the system $\Sigma_{n}$ given by (12) is $\mathcal{L}_{p}$-stable from $(W, \omega)$ to $H\left(x_{t}, \omega_{t}\right)$ for some $p \in[1, \infty]$, i.e., there exist $K_{H}, \gamma_{H} \geq 0$ such that

$$
\left\|H\left[t, t_{0}\right]\right\|_{p} \leq K_{H}\left\|x_{t_{0}}\right\|+\gamma_{H}\left\|(W, \omega)\left[t, t_{0}\right]\right\|_{p},
$$

for all $t \geq t_{0}$, and

(b) there exists $L \geq 0$ and $d: \mathbb{R} \rightarrow \mathbb{R}_{+}$, $\sup _{t \in \mathbb{R}} d(t)=\breve{d}$, such that for almost all $t \geq t_{0}$, almost all $e \in \mathbb{R}^{n_{e}}$ and for all $\left(i, x_{t}, \omega_{t}\right) \in \overline{\mathbb{N}}_{0} \times P C\left([-\bar{d}, 0], \mathbb{R}^{n_{x}}\right) \times$ $P C\left([-d, 0], \mathbb{R}^{n_{\omega}}\right)$ it holds that

$$
\begin{aligned}
\left\langle\frac{\partial W(i, e)}{\partial e}, g\left(t, x_{t}, e_{t}, \omega_{t}\right)\right\rangle \leq & L W(i, e(t-d(t)))+ \\
& +\left\|H\left(x_{t}, \omega_{t}\right)\right\| .
\end{aligned}
$$

Then, the NCS (9) is $\mathcal{L}_{p}$-stable with bias from $\omega$ to $(H, e)$ for each $\tau$ for which there exist $M>1$ and $\lambda>0$ satisfying (I), (II) and $\frac{2}{\lambda} \sqrt{M} \gamma_{H}<1$ with parameters $a=\frac{\bar{a}}{a} L$ and $c=\rho$.

Remark 1: According to Problem 1, condition (a) requires the underlying delays to be admissible, i.e., $\left\{d_{1}(t), \ldots, d_{l}(t)\right\} \in \mathcal{D}$. Condition (a) implies that the nominal system is robust with respect to intermittent information and disturbances. Condition (b) relates the current growth rate of $W(i, e)$ with its past values. As shown in Section $\mathrm{V}$, all recommendations and suggestions from [9] and [4] regarding how to obtain a suitable $W(i, e)$ readily apply because $W(i, e)$ describes the underlying UGES protocol (and not the plant-controller dynamics).

Remark 2 (Zeno-freeness): The left-hand sides of conditions (I) and (II) from Lemma 1 are nonnegative continuous functions of $\tau \geq 0$ and approach $\infty$ as $\tau \rightarrow \infty$. Also, these left-hand sides equal zero for $\tau=0$. Note that both sides of (I) and (II) are continuous in $\lambda, M, \lambda_{1}, \lambda_{2}$ and $\breve{d}$. Hence, for every $\lambda>0, \lambda_{1} \geq 0, M>1, \lambda_{2} \in(0,1)$ and $\breve{d} \geq 0$ there exists $\tau>0$ such that (I) and (II) are satisfied. Finally, since $\frac{2}{\lambda} \sqrt{M}$ is continuous in $\lambda$ and $M$, we infer that for every 


$$
\begin{aligned}
& f\left(t, x_{t}, e, \omega\right):=\left[\begin{array}{l}
f_{p}\left(t, x_{p_{t}}, g_{c_{t}}\left(t, x_{c_{t}}\right)+e_{u}(t), \omega_{p}(t)\right) \\
f_{c}\left(t, x_{c_{t}}, g_{p_{t}}\left(t, x_{p_{t}}\right)+e_{y}(t), \omega_{c}(t)\right)
\end{array}\right]=:\left[\begin{array}{l}
f_{1}\left(t, x_{t}, e, \omega\right) \\
f_{2}\left(t, x_{t}, e, \omega\right)
\end{array}\right] ; \quad h(t, e(t)):=\left[\begin{array}{l}
h_{y}(t, e(t)) \\
h_{u}(t, e(t))
\end{array}\right] \\
& g\left(t, x_{t}, e_{t}, \omega_{t}\right):=\left[\begin{array}{l}
\overbrace{\hat{f}_{c}\left(t, x_{p_{t}}, x_{c_{t}}, g_{p_{t}}\left(t, x_{p_{t}}\right)+e_{y_{t}}, g_{c_{t}}\left(t, x_{c_{t}}\right)+e_{u_{t}}, \omega(t)\right)}^{\hat{f}_{p}\left(t, x_{p_{t}}, x_{c_{t}}, g_{p_{t}}\left(t, x_{p_{t}}\right)+e_{y_{t}}, g_{c_{t}}\left(t, x_{c_{t}}\right)+e_{u_{t}}, \omega(t)\right)}-\left(\frac{\partial g_{c_{c}}}{\partial t}\right)_{t}\left(t, x_{c_{t}}\right)-\left(\frac{\partial g_{c}}{\partial x_{c}}\right)_{t}\left(t, x_{p_{t}}\right)-\left(\frac{\partial g_{p}}{\partial x_{p}}\right)_{t}\left(t, x_{p_{p_{t}}}\right) f_{1_{t}}\left(t, x_{t}, e, \omega\right)
\end{array}\right]
\end{aligned}
$$

finite $\gamma_{H}>0$ there exists $\tau>0$ such that $\frac{2}{\lambda} \sqrt{M} \gamma_{H}<1$. In other words, for each admissible $d_{i}(t), i \in\{1, \ldots, l\}$, the unwanted Zeno behavior is avoided and the proposed methodology does not yield continuous feedback that might be impossible to implement. Notice that each $\tau$ yielding $\frac{2}{\lambda} \sqrt{M} \gamma_{H}<1$ is a candidate for $\bar{\tau}$. Depending on $r, \lambda_{2}$, $\lambda$ and $M$, the maximal such $\tau$ is in fact MATI $\bar{\tau}$.

Corollary 1: Assume that the conditions of Theorem 2 hold and that $x$ is $\mathcal{L}_{p}$-detectable from $(W, \omega, H)$. Then the NCS (9) is $\mathcal{L}_{p}$-stable with bias from $\omega$ to $(x, e)$.

\section{NumericAl EXAMPLE}

The following numerical example is inspired by [13, Example 2.2.] and all the results are provided for $p=2$. Consider the following nonlinear delay plant

$$
\begin{aligned}
& {\left[\begin{array}{l}
\dot{x}_{p 1}(t) \\
\dot{x}_{p 2}(t)
\end{array}\right]=} \\
& {\left[\begin{array}{c}
-0.5 x_{p 1}(t)+x_{p 2}(t)-0.25 x_{p 1}(t) \sin \left(u(t) x_{p 2}\left(t-d_{p 1}\right)\right) \\
x_{p 1}(t) \sin \left(u(t) x_{p 2}\left(t-d_{p 1}\right)\right)+1.7 x_{p 2}\left(t-d_{p 2}\right)+u(t)-x_{p 2}(t)
\end{array}\right]+} \\
& \quad+\left[\begin{array}{c}
\omega_{1}(t) \\
\omega_{2}(t)
\end{array}\right]
\end{aligned}
$$

controlled with

$$
u(t)=-2 x_{p 1}(t)-2 x_{p 2}(t) .
$$

Apparently, $x(t):=x_{p}(t)=\left(x_{p 1}(t), x_{p 2}(t)\right)$. Additionally, $\omega(t):=\left(\omega_{1}(t), \omega_{2}(t)\right)$.

Let us consider the NCS setting in which noisy information regarding $x_{p 1}$ and $x_{p 2}$ are transmitted over a communication network while the control signal is not transmitted over a communication network nor distorted (i.e., $\hat{u}=u$ ). In addition, suppose that the information regarding $x_{p 2}$ arrives at the controller with delay $d$ while information regarding $x_{p 1}$ arrives in timely manner (i.e., $d_{p 1}=0$ ). For the sake of simplicity, let us take $d=d_{p 2}$. Apparently, the output of the plant is $y(t)=x_{p}(t)=x(t)$ and there are two NCS links so that $l=2$. Namely, $x_{p 1}$ is transmitted through one NCS link while $x_{p 2}$ is transmitted through the second NCS link. The repercussions of these two NCS links are modeled via the following error vector

$$
e=\left[\begin{array}{l}
e_{1} \\
e_{2}
\end{array}\right]=\hat{y}-(\underbrace{\left[\begin{array}{c}
x_{p 1}(t) \\
0
\end{array}\right]+\left[\begin{array}{c}
0 \\
x_{p 2}(t-d)
\end{array}\right]}_{y_{t}}) .
$$

The expressions (10) and (11) for this example become:

$$
\begin{aligned}
& \dot{x}(t)=\underbrace{\left[\begin{array}{cc}
-0.5 & 1 \\
-2 & -1
\end{array}\right]}_{A_{1}} x(t)+\underbrace{\left[\begin{array}{cc}
0 & 0 \\
0 & -0.3
\end{array}\right]}_{A_{2}} x(t-d)+ \\
& +\underbrace{\left[\begin{array}{rr}
-0.25 & 0 \\
1 & 0
\end{array}\right]}_{B_{1}} x(t) N\left(x_{t}, e\right)+\underbrace{\left[\begin{array}{cc}
0 & 0 \\
-2 & -2
\end{array}\right]}_{B} e(t)+\omega(t), \quad(17) \\
& \dot{e}(t)=\dot{\hat{y}}-B e(t-d)+\underbrace{\left[\begin{array}{cc}
0.5 & -1 \\
0 & 0
\end{array}\right]}_{C_{1}} x(t)+\underbrace{\left[\begin{array}{ll}
0 & 0 \\
2 & 2
\end{array}\right]}_{C_{2}} x(t-d)+ \\
& +\underbrace{\left[\begin{array}{cc}
0 & 0 \\
0 & 0.3
\end{array}\right]}_{C_{3}} x(t-2 d)+\underbrace{\left[\begin{array}{cc}
0.25 & 0 \\
0 & 0
\end{array}\right]}_{C_{4}} x(t) N\left(x_{t}, e\right)+ \\
& +\underbrace{\left[\begin{array}{cc}
0 & 0 \\
-1 & 0
\end{array}\right]}_{C_{5}} x(t-d) N\left(x_{t}, e_{t}\right)+\underbrace{\left[\begin{array}{rr}
-1 & 0 \\
0 & 0
\end{array}\right]}_{C_{6}} \omega(t)+\underbrace{\left[\begin{array}{cc}
0 & 0 \\
0 & -1
\end{array}\right]}_{C_{7}} \omega(t-d),
\end{aligned}
$$

where $N\left(x_{t}, e\right):=\sin \left(\left[-2\left(x_{p 1}(t)+e_{1}(t)\right)-2\left(x_{p 2}(t-d)+\right.\right.\right.$ $\left.\left.\left.e_{2}(t)\right)\right] x_{p 2}\left(t-d_{p 1}\right)\right)$ and $N\left(x_{t}, e_{t}\right):=\sin \left(\left[-2\left(x_{p 1}(t-d)+\right.\right.\right.$ $\left.\left.\left.e_{1}(t-d)\right)-2\left(x_{p 2}(t-2 d)+e_{2}(t-d)\right)\right] x_{p 2}\left(t-d_{p 1}-d\right)\right)$.

According to [9] and [4], we select $W_{R R}(i, e):=\|D(i) e\|$ and $W_{T O D}(t, e):=\|e\|$, where $D(i)$ is a diagonal matrix whose diagonal elements are lower bounded by 1 and upper bounded by $\sqrt{l}$. Next, we determine $L_{R R}, H_{R R}(x, \omega, d)$, $L_{T O D}$ and $H_{T O D}(x, \omega, d)$ from Theorem 2 for the $\mathrm{ZOH}$ strategy (i.e., $\dot{\hat{y}} \equiv \mathbf{0}_{n_{y}}$ ) as follows:

$$
\begin{aligned}
& \left\langle\frac{\partial W_{R R}(i, e)}{\partial e}, \dot{e}\right\rangle \leq\|D(i) \dot{e}\| \leq \sqrt{l}\|B\|\|D(i) e(t-d)\|+ \\
& \quad+\sqrt{l}\left(\| C_{1} x(t)+C_{2} x(t-d)+C_{3} x(t-2 d)+C_{6} \omega(t)+\right. \\
& \left.\quad+C_{7} \omega(t-d)\|+\| C_{4} x(t)\|+\| C_{5} x(t-d) \|\right)\left(x_{t}, \omega_{t}\right), \\
& \left\langle\frac{\partial W_{T O D}(i, e)}{\partial e}, \dot{e}\right\rangle \leq\|B\|\|e(t-d)\|+ \\
& \quad+\left(\| C_{1} x(t)+C_{2} x(t-d)+C_{3} x(t-2 d)+C_{6} \omega(t)+\right. \\
& \left.\quad+C_{7} \omega(t-d)\|+\| C_{4} x(t)\|+\| C_{5} x(t-d) \|\right)\left(x_{t}, \omega_{t}\right),
\end{aligned}
$$

In order to estimate $\gamma_{H}$, we utilize the following LyapunovKrasovskii functional

$$
V\left(x_{t}\right)=x(t)^{\top} C x(t)+\int_{-d}^{0} x(t+\theta)^{\top} E x(t+\theta) \mathrm{d} \theta,
$$

where $C$ and $E$ are positive-definite symmetric matrices [14, Chapter 6], [15].

Detectability of $x$ from $(W, x, H)$, which is a condition of Corollary 1 , is easily inferred by taking $x(t)$ to be the output of the nominal system and computing the respective 

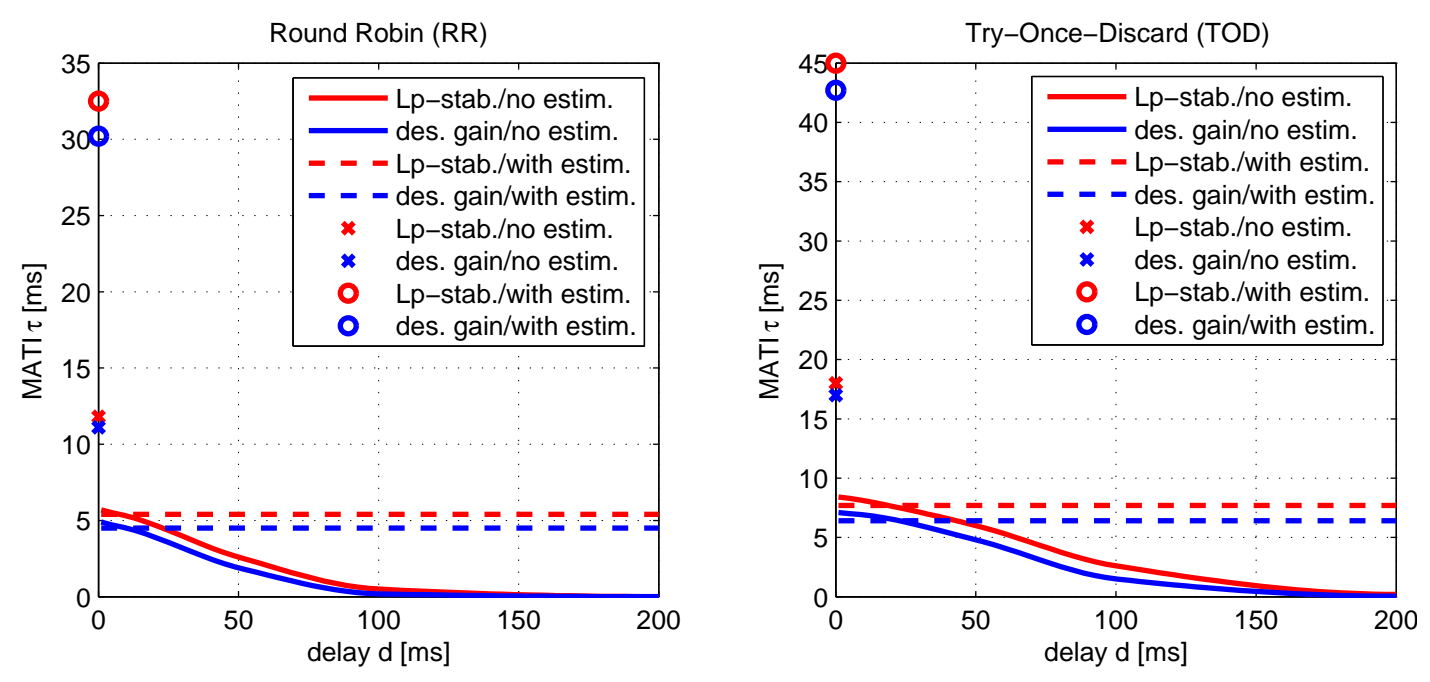

Fig. 2. Numerically obtained MATIs for different delay values $d \geq 0$ in scenarios with and without estimation.

$\mathcal{L}_{2}$-gain $\gamma_{d}$. Next, let us take the output of interest to be $x$ and find MATIs that yield the desired $\mathcal{L}_{p}$-gain from $\omega$ to $x$ to be $\gamma_{\text {des }}=50$. Essentially, the following condition

$$
\gamma_{W} \gamma_{H}<1-\frac{\gamma_{d}}{\gamma_{\text {des }}}
$$

needs to be satisfied (by changing $\gamma_{W}$ through changing MATIs) in order to achieve the desired gain $\gamma_{\text {des }}$.

Furthermore, we utilize the following estimator

$$
\begin{gathered}
\dot{\hat{y}}=B \hat{y}(t-d)=B\left(e(t-d)+\left[\begin{array}{ll}
1 & 0 \\
0 & 0
\end{array}\right] x(t-d)+\right. \\
\left.+\left[\begin{array}{ll}
0 & 0 \\
0 & 1
\end{array}\right] x(t-2 d)\right) .
\end{gathered}
$$

Figure 2 provides evidence that the TOD protocol results in greater MATIs (at the expense of additional implementation complexity/costs [10]) and that the model-based estimators significantly prolong MATIs, when compared with the ZOH strategy, especially as $d$ increases. In addition, notice that the case $d=0$ boils down to ordinary differential equation modeling so that we can employ less conservative tools for computing $\mathcal{L}_{2}$-gains. Apparently, MATIs pertaining to $\mathcal{L}_{p}$-stability from $\omega$ to $(x, e)$ are greater than the MATIs pertaining to $\mathcal{L}_{p}$-stability from $\omega$ to $x$ with $\gamma_{\text {des }}=50$.

\section{Conclusion}

In this paper, we study how much information exchange between a plant and controller can become intermittent (in terms of MATIs) such that the performance objectives of interest are still met. Our framework incorporates time-varying delays and transmission intervals that can be smaller than the delays, plants/controllers with delay dynamics, external disturbances (or modeling uncertainties), UGES scheduling protocols (e.g., RR and TOD protocols), distorted data and model-based estimators. As expected, the TOD protocol results in greater MATIs than the RR protocol. Likewise, estimation (rather than the ZOH strategy) in between two consecutive transmission instants extends the MATIs.
The future work is oriented towards devising event- and self-triggered realizations of our approach.

\section{REFERENCES}

[1] J. P. Hespanha, P. Naghshtabrizi, and X. Yonggang, "A survey of recent results in Networked Control Systems," Proceedings of the IEEE, vol. 95 , no. 1, pp. 138 - 162, January 2007.

[2] D. Tolić and S. Hirche, "Stabilizing transmission intervals and delays for nonlinear networked control systems: The large delays case," in Proceedings of the IEEE Conference on Decision and Control, Los Angeles, CA, December 2014, pp. 1203-1208.

[3] F. Mazenc, M. Malisoff, and T. N. Dinh, "Robustness of nonlinear systems with respect to delay and sampling of the controls," Automatica, vol. 49, no. 6, pp. 1925-1931, 2013.

[4] W. P. M. H. Heemels, A. R. Teel, N. V. de Wouw, and D. Nešić, "Networked Control Systems with communication constraints: Tradeoffs between transmission intervals, delays and performance," IEEE Tran. on Automatic Control, vol. 55, no. 8, pp. 1781-1796, 2010.

[5] A. Kruszewski, W. J. Jiang, E. Fridman, J. P. Richard, and A. Toguyeni, "A switched system approach to exponential stabilization through communication network," IEEE Trans. on Automatic Control, vol. 20, no. 4, pp. 887-900, 2012.

[6] B. Zhang, A. Kruszewski, and J. P. Richard, "A novel control design for delayed teleoperation based on delay-scheduled LyapunovKrasovskii functionals," International Journal of Control, vol. 87, no. 8, pp. 1694-1706, 2014.

[7] T. Estrada and P. J. Antsaklis, "Model-based control with intermittent feedback: Bridging the gap between continuous and instantaneous feedback," Int. J. of Control, vol. 83, no. 12, pp. 2588-2605, 2010.

[8] G. H. Ballinger, "Qualitative theory of impulsive delay differential equations," Ph.D. dissertation, Univ. of Waterloo, Canada, 1999.

[9] D. Nešić and A. R. Teel, "Input-output stability properties of Networked Control Systems," IEEE Transactions on Automatic Control, vol. 49, no. 10, pp. 1650-1667, October 2004.

[10] D. Christmann, R. Gotzhein, S. Siegmund, and F. Wirth, "Realization of Try-Once-Discard in wireless multihop networks," IEEE Transactions on Industrial Informatics, vol. 10, no. 1, pp. 17-26, Feb 2014.

[11] H. Khalil, Nonlinear Systems, 3rd ed. Prentice Hall, 2002.

[12] A. Anokhin, L. Berezansky, and E. Braverman, "Exponential stability of linear delay impulsive differential equations," Journal of Mathematical Analysis and Applications, vol. 193, no. 3, pp. 923-941, 1995.

[13] J. Tsinias, "A theorem on global stabilization of nonlinear systems by linear feedback," Systems \& Control Letters, vol. 17, no. 5, pp. 357-362, 1991.

[14] S. Boyd, L. El-Ghaoui, E. Feron, and V. Balakrishnan, Linear matrix inequalities in systems and control theory. Philadelphia: SIAM, 1994.

[15] D. F. Coutinho and C. E. de Souza, "Delay-dependent robust stability and $\mathcal{L}_{2}$-gain analysis of a class of nonlinear time-delay systems," Automatica, vol. 44, no. 8, pp. 2006-2018, 2008. 